\title{
Optimization and Simulation of Machining Parameters in Radial-axial Ring Rolling Process
}

\author{
Shuiyuan Tang, Jiping Lu*, Hongli Fan, Ruizhao Du \\ School of Mechanical Engineering, Beijing Institute of Technology \\ Beijing 100081, China \\ Zhonghua Jiang \\ Institute of Science \& Technology, China North Industries Group Corporation \\ Beijing 100089, China \\ Ning Ma \\ Department of Mechanical Engineering, Karlsruhe Institute of Technology \\ Karlsruhe 76131, Germany
}

Received: 15-03-2011

Accepted: 18-04-2011

\begin{abstract}
Ring rolling is a complicated process, in which rolling parameters influence directly the quality of ring. It is a process method with high productivity and few waste of material, widely used in transportation industry including automotive, shipbuilding, aerospace etc. During the rolling process of large-sized parts, crinkle and hollows often appear on surface, due to inconsistence of rolling motions with the deformation of ring part. Based on radial-axial ring rolling system configuration, motions and forces in rolling process are analyzed, and a dynamic model is formulated. Error of ring's end flatness and roundness are defined as the characteristic parameters of ring quality. The relationship between core roller feed speed, drive roller speed, the upper taper roller feed speed, and quality of ring part are analyzed. The stress and strain of the part are simulated in the Finite Element Method by DEFORM software. The simulation results provide a reference for the definition of ring rolling process parameters. It is able to make the deformation of the part be consistent with the process parameters, and improve product quality considerably.
\end{abstract}

Keywords: ring rolling, rolling parameters, forming quality.

\section{Introduction}

Ring rolling is a kind of plastic fabrication technology, which makes the wall thickness be decreased, the diameter be expanded, and the section profile be shaped by stress. ${ }^{1}$ It is a process method with high productivity and few waste of material, widely used in transportation industry including automotive, shipbuilding, aerospace, and other fields.
The forming process in radial-axial ring rolling is very complex, involving elastic/plastic deformation of ring part. The quality of fabrication is not only related to fabrication parameters, such as part material, forming temperature, forming pressure, core speed and so on, ${ }^{2,3}$ but also coincided with parameters' coordination relationship. If fabrication parameters are not harmonious, there would be defects in part such as tails and cracks. Fig. 1 shows two defect parts when speeds and stress are

*Corresponding author: jipinglu@bit.edu.cn. 
not appropriate to part forming. So it is necessary to study in detail on radial-axial ring rolling process.

Based on radial-axial ring rolling theory, we established a dynamic model of radial-axial ring rolling to analyze motions, relationships between components and rolling parameters. The result is helpful to improve the quality of rolling rings.

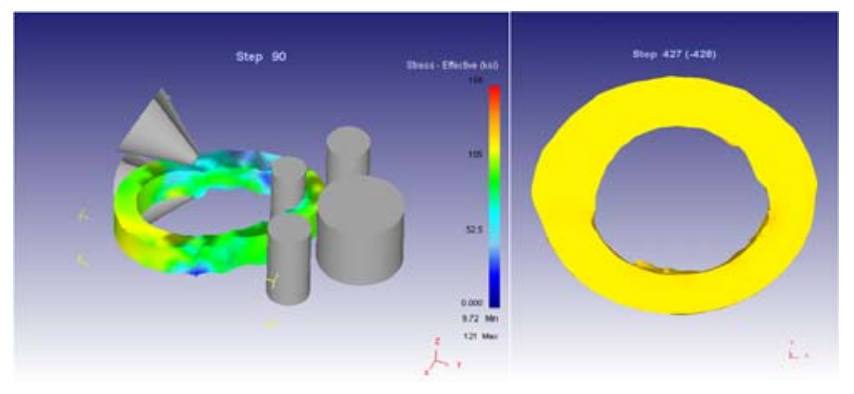

Fig. 1. R rolling System configuration and ring part with defects

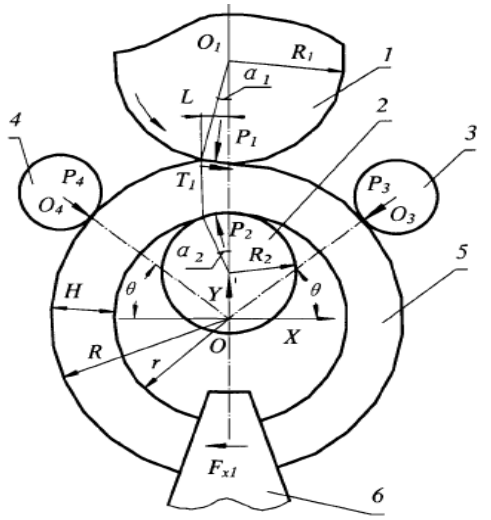

1-drive roller, 2-core roller, 3/4-guide roller, 5-ring,6-taper roller

Fig. 2. The stress analysis diagram of the ring

\section{The radial-axial ring rolling theory}

The principle of ring radial-axial rolling is shown in Fig. $2^{4}$. The ring is rotated with the friction of driven roller's rotation. The core roller always contacts the inner side of the ring, making a linear feed motion in the radial direction while rotating. The upper taper roller has a down feed motion under the function of cylinder and a rotating motion along its own axis. The lower taper roller rotates on its own axis. The guide roller always contacts the outer side of the ring, and moves backwards while the diameter of the ring is increased, and it rotates with the rotating friction of the ring. With the combination of all rollers, the radial thickness and axial height of the ring are reduced, both the inner and the outer diameter are enlarged, forming a continuous cross-section profile of local plastic deformation. It moves until the ring parameters meet the design requirements.

According to Newton's law, the ring force is balanceable, namely:

$$
\begin{aligned}
& \sum F_{\Lambda}=0 \\
& \sum F_{X}=P_{1 x}+T_{1 x}+P_{2 x}+P_{3 x}+P_{4 x}-F_{x 1} \\
& =-P_{1} \sin \frac{\alpha_{1}}{2}+\mu_{1} P_{1} \cos \frac{\alpha_{1}}{2}-P_{2} \sin \frac{\alpha_{2}}{2}-P_{3} \cos \theta+ \\
& P_{4} \cos \theta-2 \mu_{2} P \cos \frac{\beta}{2}=0 \\
& \sum F_{y}=P_{1 y}+T_{1 y}+P_{2 y}+P_{3 y}+P_{4 y} \\
& =-P_{1} \cos \frac{\alpha_{1}}{2}-\mu_{1} P_{1} \sin \frac{\alpha_{1}}{2}+P_{2} \cos \frac{\alpha_{2}}{2}-P_{3} \sin \theta-P_{4} \sin \theta=0
\end{aligned}
$$


$\mathrm{P} 1$ and $\mathrm{T} 1$ are the positive pressure and the friction of the ring by driven roller respectively, $\mathrm{P} 2$ is the positive pressure by core roller, $\mathrm{P} 3$ and $\mathrm{P} 4$ are the positive pressure by two guide rollers. Fx1 is the component stress of taper roller in radial axis, $\mathrm{P}$ is the positive pressure of ring by taper roller. al is contact angle between drive roller and the ring, a2 the contact angle between core roller and the ring. $\theta$ is the angle between the line, connecting centers of the ring and the taper roller, and the horizontal direction. $\beta$ is the frictional angle between ring and taper roller. The core roller and two guide rollers is idling, with no friction torque, and they also produce no friction force on the ring. ${ }^{5}$

During rolling progress, the drive roller and the core roller are exerted reaction force by the ring, as shown in
Fig.3, the red curve is the force in radial direction of drive roller during rolling progress, and the green curve stands for the force in radial direction of core roller. The forces upon the upper/lower taper rollers are shown in Fig.4, where the force upon the upper taper roller is much lower than that on the lower taper roller.

During rolling progress, the drive roller and the core roller are exerted reaction force by the ring, as shown in Fig.3, the red curve is the force in radial direction of drive roller during rolling progress, and the green curve stands for the force in radial direction of core roller. The forces upon the upper/lower taper rollers are shown in Fig.4, where the force upon the upper taper roller is much lower than that on the lower taper roller.

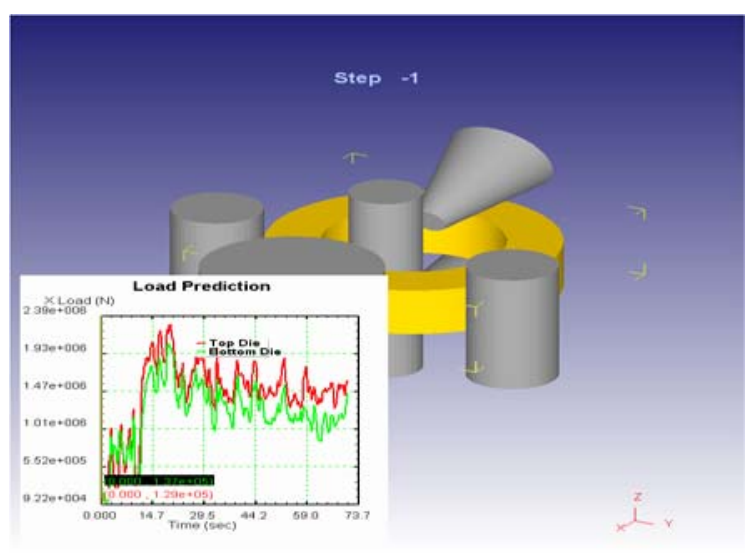

Fig. 3. The stress curve of drive roller and core roller

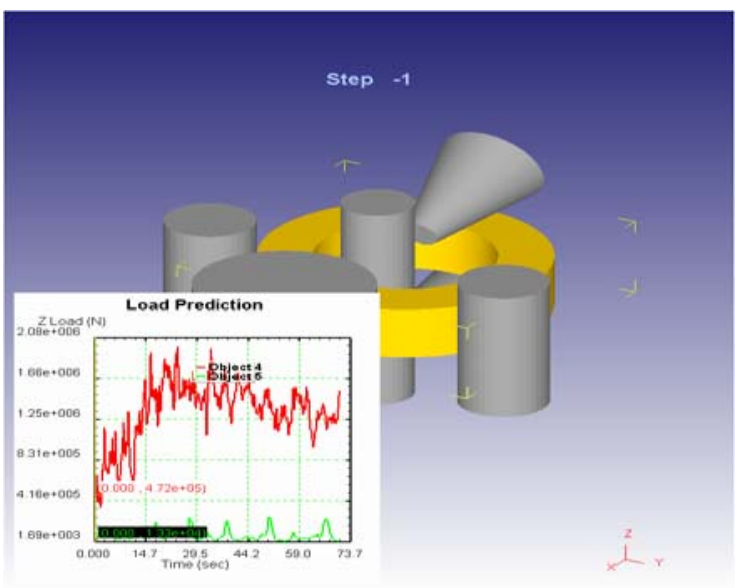

Fig. 4. The stress curve of upper cone roller 


\section{The ring rolling motion analysis}

Given, before rolling, the outer diameter of the ring is $\mathrm{R}_{0}$, the inner diameter is $\mathrm{r}_{0}$, the wall thickness is $\mathrm{b}_{0}$, the axial height is $h_{0}$. During rolling, the instantaneous value is $R, r, b, h$, respectively. $V_{D}$ is the speed of the outer diameter expandedness, $V_{d}$ the speed of the inner diameter expandedness, the feed rate of the core roller $\mathrm{V}$ is the speed of the wall thickness reduction. The relationship between parameters above is listed as the follow:

$$
\begin{aligned}
& v_{D}=\left(\frac{D_{0}+d_{0}}{2} \cdot \frac{H_{0}}{H^{2}}-1\right) v \\
& v_{d}=v_{D}+2 v \\
& v=-\frac{d H}{d t}
\end{aligned}
$$

Accordingly, the ring diameter is associated with the feed rate of the core roller. Only when the rate of inner diameter change is greater than the outer diameter, the wall thickness decrease can be guaranteed during the rolling. During rolling, the ring is continuously rotating, $\mathrm{n}_{1}$ and $\mathrm{R}_{1}$ represent the speed and radius of the drive

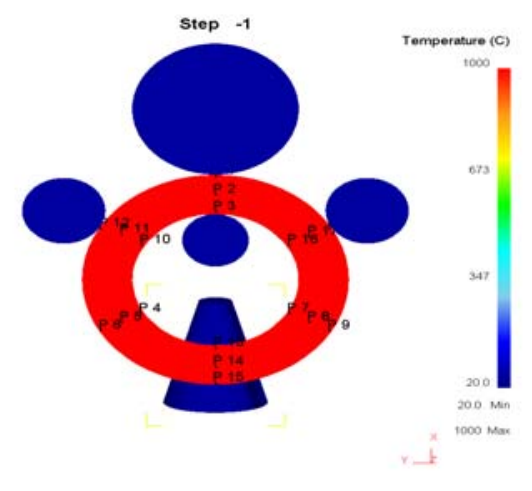

Fig. 5. The distributed points on the ring's end face

\section{Quality of ring forming}

\subsection{Quality definition}

The quality of ring forming in rolling progress is defined by the flatness of the end face and the roundness roller respectively. $\mathrm{R}$ and $\mathrm{n}$ are the instantaneous speed and outer diameter of the ring. If the relative sliding between the ring and the drive roller is ignored, the speed of the ring rotation is carried out as the follow:

$$
n=\frac{2 n_{1} R_{1}}{\frac{D_{0}+d_{0}}{2} \frac{H_{0}}{H}+H}
$$

During the rolling, the ring center gradually retreats, and its speed $\mathrm{V}_{0}$ is equals half of the outer-diameter-increase speed, ${ }^{6}$ namely:

$$
V_{o}=\frac{1}{2} V_{D}=\frac{1}{2}\left(\frac{D_{0}+d_{0}}{2} \frac{H_{0}}{H^{2}}-1\right) V
$$

The core roller feeds in a straight line, and is rotated by the friction between the drive roller and the ring. The rotate speed of the core roll is:

$$
n_{2}=\frac{d_{0}}{d_{2}} n
$$

The line feed speed is:

$$
v=\frac{n_{1} R_{1} \Delta h}{R}
$$

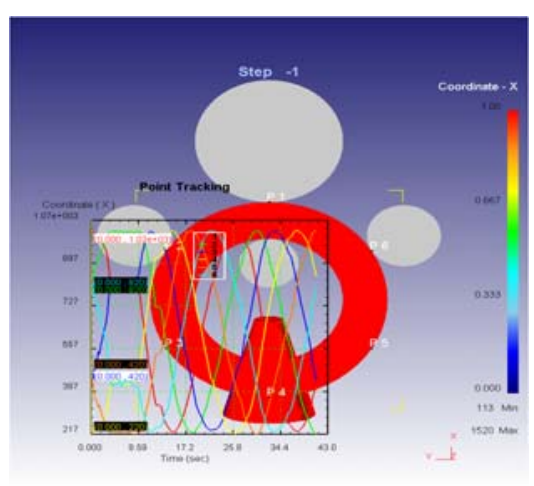

Fig. 6. The distributed points on the ring's excircle

error of the ring's excircle. ${ }^{7}$ Selected 18 points on the end face as shown in Fig.5, the flatness of the end face is calculated by the vertical coordinates to evaluate the surface quality of the ring end face. According to the flatness, the forming quality of ring is analyzed under different feed rates. Selected 6 points as shown in Fig.6, 
a basic circle is calculated by the least square method, and the roundness error is also calculated.

The base circle equation is $x^{2}-2 A x+y^{2}-2 B y-C=0$, according to the least square, the deviation value is calculated as the following:

$$
\begin{aligned}
& A \sum_{i=1}^{n} 2 x_{i}^{2}+B \sum_{i=1}^{n} 2 x_{i} y_{i}+C \sum_{i=1}^{n} x_{i}=\sum_{i=1}^{n} x_{i} z_{i} \\
& A \sum_{i=1}^{n} 2 x_{i} y_{i}+B \sum_{i=1}^{n} 2 y_{i}^{2}+C \sum_{i=1}^{n} y_{i}=\sum_{i=1}^{n} y_{i} z_{i} \\
& A \sum_{i=1}^{n} 2 x_{i}+B \sum_{i=1}^{n} 2 y_{i}+C n=\sum_{i=1}^{n} z_{i}
\end{aligned}
$$

The result of the roundness error is:

$$
\delta=\max \left\{\sqrt{\left(x_{i}-x_{0}\right)^{2}+\left(y_{i}-y_{0}\right)^{2}}\right\}-\min \left\{\sqrt{\left(x_{i}-x_{0}\right)^{2}+\left(y_{i}-y_{0}\right)^{2}}\right\}
$$

The simulation results demonstrate that with the increasing of the feed rate of the mandrel, the effective strain of the ring is decreasing and the surface quality is improving. ${ }^{8}$ With faster feed rate, the width of the ring internal diameter and the outside diameter is decreased significantly, however, the mid-diameter width of the ring is increased, the quality of the end face and the ring surface is improved. With the slower feed rate, the widths of the ring's internal diameter and outside diameter are increased distinctly, and the width of the middle diameter becomes small. Some crow's-feet appear obviously in the free end face on the ring, and the convex-concave are produced. The reason is that the feed rate is proportional to each rotation feed. With the increase of the feed rate, each rotation feed is improved, and the whole ring is penetrated with yield region, which is beneficial to deformation of the ring spread in circumference and the thickness diminution.

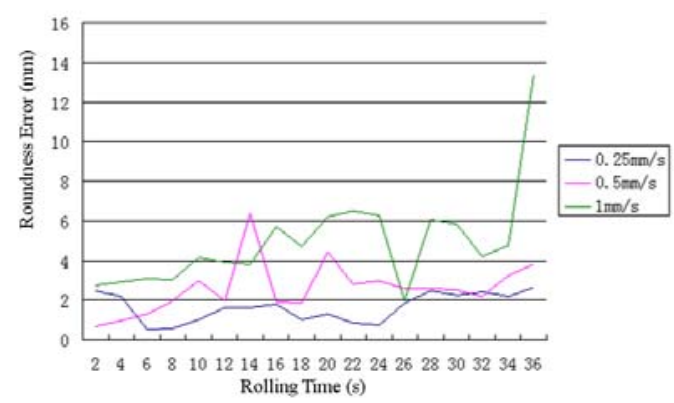

Fig. 7. The relation curves between feed speed and the roundness error

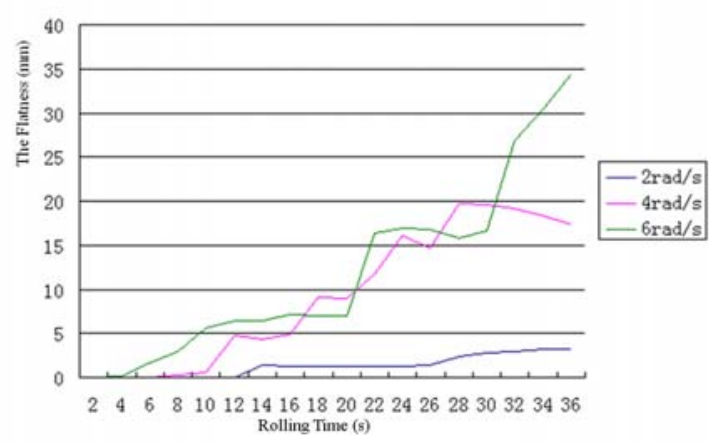

Fig. 8. The relation curves between the feed speed and the end face flatness 


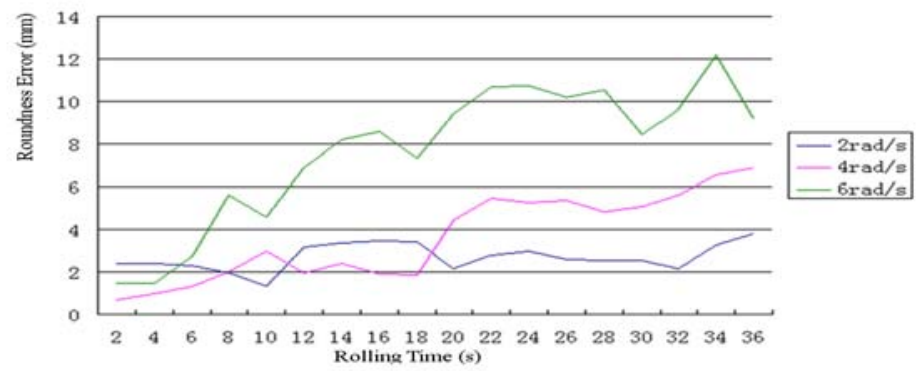

Fig. 9. The relation curves between the speed of the drive roller and the roundness error

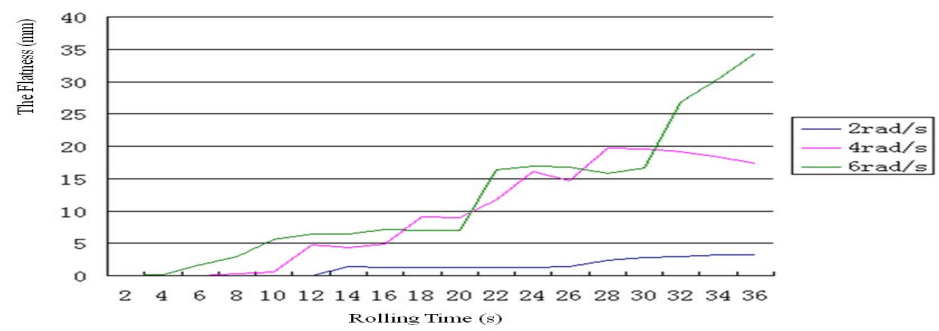

Fig. 10. The relation curves between the speed of the drive roller and the end face flatness

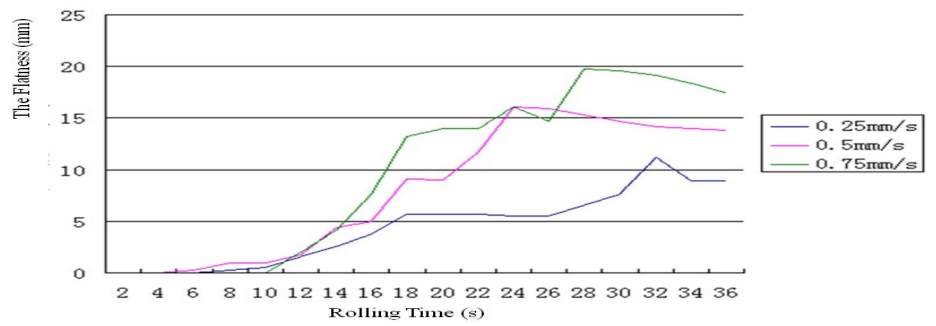

Fig. 11. The relation curves between the feed speed of the taper roller and the end face flatness

\subsection{Relationship of the forming rate and quality}

With the slower feed rate, the ring moves less numbers of turns. So yield region during rolling process mainly concentrates on the ring rolling surface. The deformation of mid-diameter is smaller, deformation region at axis-direction is more inhomogenous, which leads to width deformation on excircle/internal surface. ${ }^{9}$ Fig.7 and Fig. 8 show the roundness error and the surface quality curve at the feed rate of $0.25 \mathrm{~mm} / \mathrm{s}, 0.5 \mathrm{~mm} / \mathrm{s}$ and $1 \mathrm{~mm} / \mathrm{s}$ respectively.

When the drive roller moves at low speed, the number of the ring rotation is reduced within the same rolling time, resulting in pre and post forming, and the rolling of the ring becomes difficult. ${ }^{9}$ The high speed of the drive roller is conducive to ring forming, and the contact area between core roller and the ring per unit of time increases between the inside roller and the ring. Fig. 9 shows the relation between the roundness error and the drive roller speed. According to the simulation curve, a conclusion can be drawn that the higher the speed is, the faster the material is deformed. The relation between the drive roller speed and the flatness is shown in Fig.10. It shows that the flatness value increases with the feed speed. 
The relation between the ring quality and the feed speed of the taper roller is simulated as shown in Fig. $11^{10}$. Fig. 12 shows the relation between the feed speed of the taper roller and the roundness error. The simulation re-

\section{Simulation of the ring rolling}

Based on the analysis of the ring rolling motion and parameters, the rolling process can be simulated with FEM software DEFORM-3D, and the stress and strain of the ring is analyzed ${ }^{11}$. During the simulation, the rotate speed of the driven roller is $1.3 \mathrm{~m} / \mathrm{s}$, the feed rate of the core roller is $2 \mathrm{~mm} / \mathrm{s}$, the ring material is steel $\mathrm{S} 45 \mathrm{C}$, the grid number is 10000 , the step length is 5000 , the constant shear friction factor is 0.7 , the initial temperature is $1200^{\circ} \mathrm{C}, \mathrm{HTC}($ Heat Transfer Coefficient) is sult shows that with the increase of the feed speed the quality of the end face flatness and the roundness is decreased.

$0.02 \mathrm{~N} /\left(\mathrm{S} \cdot \mathrm{mm}^{\circ} \mathrm{C}\right)$.As illustrated in Fig. 13 , the ring inner stress is increasing with the ring rolling. The stress is mainly concentrated on one place, where the ring contacts with the drive and taper rollers. The stress of the other area is little and uniform. As shown in Fig.14, the ring strain is generating gradually. The maximum strain takes place at the area where the ring contacts with the roller. The strain of the ring's inner wall is greater than that of the outer wall. The end face of the part is flat, while the cylinder surface is smooth.

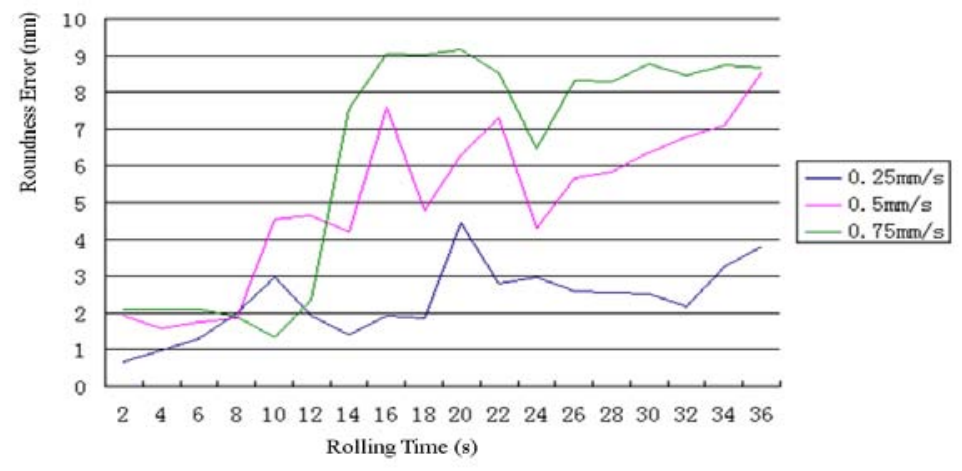

Fig. 12. The relation curves between the feed speed of the taper roller and the roundness error

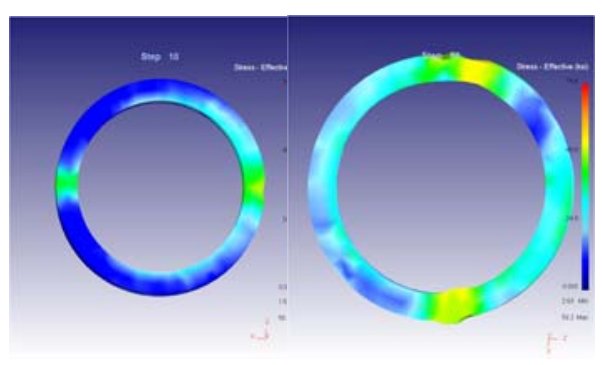

Fig. 13. The stress distribution of rolling stage

\section{Conclusions}

As we discussed above that rolling quality is defined by the end face flatness and the roundness error. When the speed of the drive roller and the feed speed become

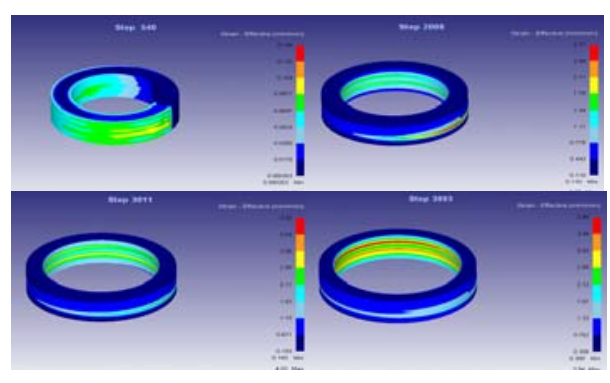

Fig. 14. The strain distribution of rolling stage

large, the error of the end faces flatness and the roundness increase. Only when the machining parameters are consistent with the deformation of the ring, would the product be qualified. 


\section{References}

1. L. Hua, X.G. Huang, C.D. Zhu, The Ring rolling theory and technology (Beijing, China Machine Press, 2001).

2. R.D. Jiang, Y.G. Wang, Ring rolling process modeling, Chinese Mechanical Engineering, 4(3) (1993) 6-9.

3. L.B. Pan, The research of radial-axial ring rolling deformation and CAPP System (Wuhan University of Technology, Wuhan, 2007).

4. L. Hua, Vibration and controlling rolling process, Trans. Nonferrous Met. Soc. (China), 9(2) (1999) 213-217.

5. DY Yang, JS Ryoo, JC Chui, W Johnson, Analysis of roll torque in profile ring rolling of $\mathrm{L}$-section in process, 21th Int. MTDR Conf. (London, 1981), 69-74.

6. SW Sloan, PW Klee., Upper boundary limit analysis with discontinuous velocity fields, Computer Methods in Applied Mechanics and Engineering, 127(1995) 293-314.
7. JH Lee, YH Kim., A study on flashless precision forging by the upper bound elemental technique, Journal of Material Processing Technology, 72(1997) 371-379.

8. Lim T,Pillinger I,Hartley P., A finite-element simulation of profile ring rolling using a hybrid mesh model, Journal of Processing Technology, 80-81(1998) 199-200.

9. Z.M.Hu, I.Pillinger., Three-dimension finite element modeling of ring rolling, Journal of Materials Processing Technology, 1994.

10. Alex Assenza, Maurizio Valle, Michel Verleysen, A Comparative Study of Various Probability Density estimation Methods for Data Analysis, International Journal of Computational Intelligence Systems, Vol.1, No.2, (June 2008), 188-201

11. J.L.Song, A.L.Dowsona, M.H.Jacobsa., Coupled thermomechanical finite element modeling of hot ring process, Journal of Materials Processing Technology, 121(2002) 332-34 